\title{
Politique et brevets d'inventions : les relations entre les colonies d'Amérique du Nord britannique et les États-Unis (1852-1872)
}

\section{Denis Veilleux}

Volume 48, numéro 1, été 1994

URI : https://id.erudit.org/iderudit/305300ar

DOI : https://doi.org/10.7202/305300ar

Aller au sommaire du numéro

Éditeur(s)

Institut d'histoire de l'Amérique française

ISSN

0035-2357 (imprimé)

1492-1383 (numérique)

Découvrir la revue

Citer cet article

Veilleux, D. (1994). Politique et brevets d'inventions : les relations entre les colonies d'Amérique du Nord britannique et les États-Unis (1852-1872). Revue d'histoire de l'Amérique française, 48(1), 57-84. https://doi.org/10.7202/305300ar
Résumé de l'article

Les lois concernant les brevets d'inventions au Canada, entre 1824 et 1872, ont été au centre d'une lutte entre les partisans du protectionnisme et ceux du libéralisme en matière de brevets. Ce sont leurs conceptions différentes à propos du rôle de ces lois dans le développement technologique et économique qui étaient à la source de leur affrontement.

En début de période, le protectionnisme en matière de brevets domine : seuls les Canadiens peuvent obtenir des brevets. Mais rapidement, les forces en faveur du libéralisme, stimulées par certains événements, commencèrent à exercer des pressions en faveur d'une libéralisation, c'est-à-dire permettre aux inventeurs étrangers d'obtenir des brevets canadiens.

Les forces libérales étaient autant canadiennes qu'étrangères et, dans ce dernier cas, surtout américaines. Au fur et à mesure que leurs pressions se multiplièrent et que les circonstances leur devinrent favorables, elles forcèrent les gouvernements canadiens à abandonner leurs positions protectionnistes. Dans ce contexte, les inventeurs canadiens firent progressivement coïncider leurs intérêts avec ceux des inventeurs étrangers et exigèrent une libéralisation.
Ce document est protégé par la loi sur le droit d'auteur. L'utilisation des services d’Érudit (y compris la reproduction) est assujettie à sa politique d'utilisation que vous pouvez consulter en ligne.

https://apropos.erudit.org/fr/usagers/politique-dutilisation/ 


\title{
POLITIQUE ET BREVETS D'INVENTIONS: LES RELATIONS ENTRE LES COLONIES D'AMÉRIQUE DU NORD BRITANNIQUE ET LES ÉTATS-UNIS $(1852-1872)^{1}$
}

\author{
DENIS VEILLEUX \\ Département d'histoire \\ Université McGill
}

\section{RÉSUMÉ}

Les lois concernant les brevets d'inventions au Canada, entre 1824 et 1872, ont été au centre d'une lutte entre les partisans du protectionnisme et ceux du libéralisme en matière de brevets. Ce sont leurs conceptions différentes à propos du rôle de ces lois dans le développement technologique et économique qui étaient à la source de leur affrontement.

En début de période, le protectionnisme en matière de brevets domine: seuls les Canadiens peuvent obtenir des brevets. Mais rapidement, les forces en faveur du libéralisme, stimulées par certains événements, commencèrent à exercer des pressions en faveur d'une libéralisation, c'est-à-dire permettre aux inventeurs étrangers d'obtenir des brevets canadiens.

Les forces libérales étaient autant canadiennes qu'étrangères et, dans ce dernier cas, surtout américaines. Au fur et à mesure que leurs pressions se multiplièrent et que les circonstances leur devinrent favorables, elles forcèrent les gouvernements canadiens à abandonner leurs positions protectionnistes. Dans ce contexte, les inventeurs canadiens firent progressivement coïncider leurs intérêts avec ceux des inventeurs étrangers et exigèrent une libéralisation.

\section{ABSTRACT}

Between 1824 and 1872, Canadian patent laws were the object of a struggle opposing the partisans of protectionism in the matter of patents and those of liberalism. The conceptions of the two camps about the role of these laws in technological and economic development confronted each other.

1. Cet article est tiré de notre mémoire de maîtrise, Protectionnisme et libéralisme: la lutte pour la libéralisation de la loi des brevets d'inventions au Canada (1824-1872), Université du Québec à Montréal, 1992, 172 pages. Je tiens à remercier mon directeur de mémoire, Yves Gingras, pour ses commentaires. 
At the beginning of the period, protectionism was dominant: only Canadians could be granted patents of invention in Canada. Aided by the stimulus of certain events, the partisans of liberalism began quite early to press for a liberal patent law, a law by which Canadian patents could be granted to foreign inventors.

The liberal forces were both Canadian and foreign and in the latter case especially American. As they multiplied their pressures and as the situation turned to their advantage, Canadian governments had to retreat from their protectionist stances. In that context, Canadian inventors progressively took their place beside foreign ones and insisted that the patent law be liberalized.

Les brevets d'inventions sont essentiellement des droits exclusifs accordés aux inventeurs. Ces droits -- exclusivité de fabrication, de vente, etc. - compensent pour les investissements que demande la mise au point des inventions. Par contre, pour obtenir des brevets, les inventeurs doivent soumettre spécifications et dessins afin de rendre disponibles au public intéressé les nouvelles connaissances techniques ${ }^{2}$.

De nombreux travaux d'histoire, particulièrement ces dernières années, se sont attardés sur les implications sociales et économiques des brevets d'inventions. Les sujets sont très diversifiés, allant de la diffusion des techniques à la stimulation de l'innovation technologique - ou plus généralement du développement économique en passant par la pertinence de l'utilisation des brevets comme instrument de mesure - de l'innovation ou du développement économique - ou encore touchant le fonctionnement des institutions chargées de l'octroi des brevets ${ }^{3}$.

Cet article porte sur les débats politiques soulevés par les lois sur les brevets d'inventions, et sur ce sujet, la contribution canadienne reste encore aujourd'hui relativement modeste. La thèse de J. J. Brown, à l'effet que l'histoire des inventeurs canadiens est celle de l' «exode des cerveaux» vers les États-Unis, est importante car elle soulève la question des relations entre pays de «premier rang» et pays de «second rang». Ces relations sont aussi importantes, sur le plan de l'invention et de l'innovation, pour Dianne Newell. Cette dernière souligne que les techniques minières en Ontario, dans la deuxième

2. Pour plus de détails, voir Harold Fox, Monopolies and Patents. A Study of the History and the Future of the Patent Monopoly (Toronto, University of Toronto Press, 1947) et C. H. Greenstreet, F. Liebesny, ed., «History of Patent Systems», Mainly on Patents. The Use of Industrial Property and Its Literature (Hamden, Conn., Archon Books, 1972), 1-18. Pour un aperçu synthétique, voir Bertrand Gille, Histoire des techniques (Paris, Gallimard, 1978).

3. La liste des travaux pertinents est si considérable qu'il n'est pas possible d'en faire état ici. Le lecteur intéressé trouvera de nombreuses références dans une récente livraison de la revue Technology and Culture entièrement consacrée à ce sujet; voir Technology and Culture, 32,4 (octobre 1991). Voir également les notes qui suivent. 
moitié du dix-neuvième siècle, ont d'abord dues être importées d'Angleterre et des États-Unis avant d'être adaptées aux conditions locales $^{4}$. R. T. Naylor a discuté de ces mêmes relations, mais plus particulièrement à propos des lois sur les brevets d'inventions. Essentiellement, cet auteur soutient que les différentes lois adoptées au Canada au dix-neuvième siècle ont accru la dépendance de l'économie canadienne envers les États-Unis en accentuant la dépendance technologique ${ }^{5}$.

Or au dix-neuvième siècle, les partisans des lois sur les brevets croyaient stimuler par celles-ci le développement technologique et, par là, économique. Mais ces lois avaient également leurs détracteurs et aux États-Unis comme en Europe, des débats à propos de l'incidence des brevets sur le développement économique ont opposé les deux camps $^{6}$. Au Canada ${ }^{7}$, les lois des brevets ont aussi provoqué des débats sur cette question. Mais une de leurs préoccupations majeures était justement de savoir qui pourrait ou devrait obtenir des brevets canadiens et, par conséquent, qui serait l'agent du développement technologique et économique ${ }^{8}$. Les relations entre le Canada et ses principaux partenaires sont donc, encore là, au centre des enjeux.

4. J. J. Brown, Ideas in Exile. A History of Canadian Invention (Montréal, McClelland and Stewart Limited, 1967). Dianne Newell, Technology on the Frontier, Mining in Old Ontario (Vancouver, University of British Columbia Press, 1989); “'All in a Day's Work': Local Invention on the Ontario Mining Frontier", Technology and Culture, 26,4 (octobre 1985): 799-814.

5. R. T. Naylor, The History of Canadian Business, 1867-1914 (Toronto, James Lorimer \& Company Publishers, 1975), II: 38-68.

6. Voir V. M. Batzel, «Legal Monopoly in Liberal England: the Patent Controversy in the Mid-Nineteenth Century», Business History, 22,2 (juillet 1980): 189-202. Fritz Machlup et Edith T. Penrose, «The Patent Controversy in the Nineteenth Century», Journal of Economic History, 10,1 (mai 1950): 1-29. Eric Schiff, Industrialization Without National Patents. The Netherlands, 1869-1912. Switzerland, 1850-1907 (Princeton, Princeton University Press, 1971). Pour un aperçu général des conflits, pour les cas anglais et américain, voir Christine McLeod, Inventing the Industrial Revolution. The English Patent System, 1660-1800 (Cambridge, Cambridge University Press, 1988); H. I. Dutton, The Patent System and Incentive Activity During the Industrial Revolution 1750-1852 (Manchester, Manchester University Press, 1984); William B. Bennett, The American Patent System. An Economic Interpretation (Bâton Rouge, Louisiana State University Press, 1943); et Floyd L. Vaughan, The United States Patent System. Legal and Economic Conflicts in American Patent History (Norman, University of Oklahoma Press, 1956). L'excellent ouvrage de Schiff traite du rôle et de la place des systèmes de brevets dans l'orientation du développement économique en fonction des rapports entre pays de premier rang et pays de deuxième rang.

7. Pour cet article, les termes «Canada» et «canadien» désignent l'ensemble des colonies qui adhérèrent à la Confédération en 1867. Il faut remarquer que les dates importantes de l'histoire des lois des brevets d'inventions ne correspondent pas toujours aux dates de l'histoire politique ou constitutionnelle canadienne.

8. Il est certain que ce n'est pas la seule question à avoir soulevé des débats. D'ailleurs, en analysant les différentes lois, on peut voir que ce qui pouvait être breveté, la durée des brevets, le processus de règlement des litiges, etc., a changé tout au long de la période concernée ici et même jusqu'à aujourd'hui. Cependant, l'abondance de la matière qui sert de base à cet article laisse supposer qu'il traite d'une question jugée capitale. 
Ainsi, la période 1852-1872 fut, au Canada, le théâtre d'une lutte acharnée entre deux tendances en matière de brevets. Une première tendance peut être qualifiée de libérale parce qu'elle cherchait à faire adopter des lois qui permettraient à tous les inventeurs d'obtenir des brevets canadiens, quel que soit leur pays de résidence. La deuxième, qualifiée ici de protectionniste, préconisait au contraire que seuls les Canadiens puissent jouir de ce droit ${ }^{9}$.

Cet article tente de retracer les forces sociales, les événements et les conjonctures qui ont mené à l'adoption de l'Acte concernant les Brevets d'inventions de 1872, une loi ayant des caractéristiques libérales. La loi britannique de 1852 sur les brevets d'inventions, celle des États-Unis adoptée en 1861, la Confédération, ainsi que la possibilité du renouvellement du Traité de réciprocité canado-américain sont autant de facteurs qui ont joué en faveur ou en défaveur de la libéralisation des lois canadiennes sur les brevets.

\section{LES CONSÉQUENCES AU CANADA DE LA LOI BRITANNIQUE DE 1852 SUR LES BREVETS D'INVENTIONS}

Au début de 1852, chacune des provinces qui allaient former la Confédération en 1867 avait sa propre loi sur les brevets d'inventions ${ }^{10}$. Les lois de la Nouvelle-Écosse et de la Province du Canada avaient un caractère protectionniste: dans les deux cas, seuls les résidants et sujets britanniques pouvaient obtenir des brevets ${ }^{11}$. Au Nouveau-Brunswick, par contre, la loi était plutôt libérale depuis environ un an: presque toutes les personnes intéressées pouvaient obtenir un brevet ${ }^{12}$. Quant aux États-Unis, depuis l'adoption de la loi de 1836, la législation sur les brevets était également restrictive: les

9. Du point de vue de l'économie ou de l'économie politique, il peut être hasardeux d'opposer de telles tendances. Cependant, comme l'article le montrera, il est clair qu'à l'époque les acteurs concernés s'opposeront sur le plan politique (tant par leurs arguments, leurs actions que par les lois qu'ils défendront ou condamneront). Ainsi, on peut bien, sur le plan de l'économie, discuter et remettre en question les différences entre ces tendances, mais il est indéniable que, sur le plan politique, elles aient existé. Par contre, cet article ne permettra pas de délimiter précisément les tendances politiques, en fonction des partis politiques par exemple. Cela nécessiterait un travail complémentaire.

10. Pour plus de détails sur l'évolution des lois des brevets au Canada, à partir des débuts en 1824 jusqu'en 1852, voir Margaret Coleman, «The Canadian Patent Office from Its Beginning to 1900", APT Bulletin, 8,3 (1976): 56-63; Gordon Asher, «The Development of the Patent System in Canada since 1767», Canadian Patent Recorder, 43 (1965): 56-75; et Denis Veilleux, op. cit., chap. 1.

11. Pour la Province du Canada, 12 Vict. c. 24 et pour la Nouvelle-Écosse, 14 Vict. c. 20. Dans la Province du Canada, il est possible d'obtenir des brevets sur des inventions étrangères; c'est pourquoi certains intervenants parlent de pillage.

12. 14 Vict. c. 35 . 
inventeurs étrangers devaient payer des tarifs beaucoup plus élevés que les inventeurs américains ${ }^{13}$.

Bien que le protectionnisme dominait en 1852, il n'en existait pas moins depuis plusieurs années un courant favorable à la libéralisation en matière de brevets, tant au Canada qu'aux États-Unis. On peut voir ce courant de pensée dans certains rapports annuels du commissaire des brevets des États-Unis ${ }^{14}$. Au Canada, il est présent dans divers projets de loi publique ${ }^{15}$. Quelques projets de loi privée ont également été présentés devant les différentes législatures afin, soit de répondre à des cas spéciaux - une invention faite par un inventeur local associé à un inventeur étranger par exemple ${ }^{16}$-, soit pour contourner les lois protectionnistes ${ }^{17}$. De plus, entre 1840 , moment de la création de la Province du Canada, et l'adoption de sa première loi sur les brevets d'inventions en 1849, plusieurs demandeurs ou détenteurs de brevets ont cherché à faire étendre ceux-ci à l'ensemble de la province même si les lois en vigueur pendant cette période ne le permettaient pas ${ }^{18}$.

13. Act of 1836, chapter 357. La loi américaine de 1832 était plus libérale. Il ne faut pas s'étonner du changement opéré en 1836 lorsque l'on sait qu'en 1833 et 1834 respectivement, la Nouvelle-Écosse et le Nouveau-Brunswick suivent le Bas et le Haut-Canada et adoptent leurs premières lois des brevets qui sont, elles aussi, protectionnistes.

14. Voir Annual Reports of the Commissioner of Patents (Washington, 1843-1852).

15. Deux projets sont présentés entre 1824 et 1852: celui de William Badgley en 1849 et celui de Narcisse-Fortunat Belleau en 1852, tous deux à l'Assemblée législative de la Province du Canada. Voir Debates of the Legislative Assembly of United Canada (DLAUC) (1849), 12, 48, 406, 936-937, 1721, 1976, 2007, 2243 et 2502; Journals of the Legislative Assembly of the Province of Canada (JLAPC) (1849), 13, 57, 105, 211, 249, 253, 293 et 364 et Journals of the Legislative Council of the Province of Canada (JLCPC), 7 et 11 juin 1852.

16. Nous ne mentionnerons ici que les noms des demandeurs et l'année des demandes: James Radcliffe en 1827, Thomas Hornor en 1830 au Haut-Canada, John Counter en 1850 dans la Province du Canada. Ces informations ont été tirées des Journaux et des Débats des assemblées et des conseils législatifs des provinces concernées.

17. Il y a, par exemple, le cas de Thomas Robson, de Sackville au Nouveau-Brunswick. Ce dernier fera de nombreuses demandes de brevets, entre mars 1850 et mai 1853 aux législatures de la Nouvelle-Écosse et de la Province du Canada pour une invention, une corme de brume, pour laquelle il avait obtenu un brevet dans sa province de résidence. Ces informations ont été tirées des Journaux et des Débats des Assemblées et des Conseils législatifs des provinces concernées.

18. En effet, entre 1840 et 1849 , il semble que les anciennes lois du Bas-Canada et du Haut-Canada aient continué à être appliquées. Dans la liste des brevets accordés par chacune des sections de la Province du Canada durant cette période, on peut voir que certains inventeurs ont réussi à obtenir des brevets dans les deux sections pour la même invention. Voir List of Canadian Patents, from the Beginning of the Patent Office, (June, 1824) (Ottawa, Gordon Publications \& Reproductions, 1979). De plus, dans les archives des secrétaires provinciaux, on peut noter que certains demandeurs de brevets s'informaient de la possibilité de faire étendre leurs brevets aux deux sections. Certains inventeurs l'exigeaient. En 1851, une loi de la Province du Canada prévoit que les brevets du Bas et du Haut-Canada toujours valides pouvaient être étendus à l'ensemble de la Province; voir 14 \& 15 Vict. c. 79. 
Finalement, avant 1852, plusieurs inventeurs ont obtenu pour la même invention, des brevets de deux provinces ou plus ${ }^{19}$.

Les tensions entre protectionnisme et libéralisme seront exacerbées par l'adoption, en 1852, de la loi britannique qui ne permet plus d'étendre la validité des brevets anglais dans les colonies ayant une loi empêchant une telle expansion ${ }^{20}$, ce qui est le cas pour les provinces de l'Amérique du Nord britannique (ANB). Il est difficile de dire si des inventeurs se sont prévalus de leur droit de faire étendre leurs brevets britanniques en ANB, mais il n'en reste pas moins que la loi de 1852 vient leur enlever cette possibilité. Cela pouvait leur apparaître d'autant plus important que les provinces de l'ANB étaient entrées dans une phase d'industrialisation.

D'ailleurs, dès le début de 1853 , après que des inventeurs probablement anglais - en aient exprimé le souhait, le gouvernement britannique enquête auprès des autorités coloniales de l'ANB pour savoir s'il ne serait pas possible d'y faire étendre la validité des brevets britanniques ${ }^{21}$. Nous ne savons pas quelle fut la réponse officielle des colonies de l'ANB, mais en Nouvelle-Écosse certains individus ont été favorables à cette possibilité ${ }^{22}$. Rien n'a cependant été fait à cet égard ni en Nouvelle-Écosse ni dans la Province du Canada.

Par contre, au mois d'avril 1853, un pas vers le libéralisme en matière de brevets est quand même franchi en Nouvelle-Écosse. Les sujets britanniques et résidents depuis plus d'un an dans la Province

19. En consultant les listes de brevets octroyés dans les différentes colonies entre 1824 et 1852 , on peut repérer un certain nombre d'individus qui ont obtenu des brevets pour deux colonies ou plus pour la même invention. En plus de Robson, c'est le cas de B. F. Tibbits, W. S. Cleveland, Z. Lane, J. Levi Rice, J. Ross, William Watts, George Hawley et Asa Willard. Voir List of Canadian Patents (...); Lovell's Canadian Dictionary for 1871 (...) (Montréal, J. Lovell, [1871]), 2325-2356; Patents of Canada from 1824 to 1849 (Toronto, 1860); Patents of Canada from 1849 to 1855 (Toronto, 1860) et ANC, MG9 A1, New Brunswick, Executive Council, Patents of Invention, vol. 123, finding aid no. 121.

20. 15-16 Vict. c. 83; voir également Dunbar Browne, «The History and Law of Letters Patent of Inventions", Journal of the Board of Arts and Manufactures for Upper Canada (JBAMUC), 1 (1861): 265. La loi de 1852 permettait aux étrangers d'obtenir des brevets anglais. Avant 1852, le système des brevets reposait sur le Statut des Monopoles de 1624 et la jurisprudence que son application avait entrainée. Si le Statut des Monopoles visait à limiter l'octroi de privilèges exclusifs par la couronne, il ne lui perrnettait pas moins d'en accorder pour l'établissement de nouvelles «manufactures» dans le royaume. Plusieurs historiens ont souligné que l'intention derrière l'adoption de ce Statut était, entre autres, d'attirer les artisans et les inventeurs étrangers à venir s'établir en Angleterre pour y introduire de nouvelles «manufactures». Selon cette analyse, avant 1852 , les étrangers pouvaient donc obtenir des brevets anglais. Le Statut des Monopoles resta en vigueur avec peu de modifications jusqu'en 1852. Voir Christine McLeod, op. cit., H. I. Dutton, op. cit., et Harold Fox, op. cit.

21. ANC, RG7 G1 vol. 131, 1, et Journals of the House of Assembly of Nova Scotia (JHANS) (1853), appendice 40.

22. Idem. 
du Canada, de l'île-du-Prince-Édouard, du Nouveau-Brunswick et de Terre-Neuve, peuvent désormais, au moment de leur demande, obtenir des brevets de la Nouvelle-Écosse ${ }^{23}$. Le protectionnisme de cette colonie prend donc les allures d'un protectionnisme canadien, et on pourrait dire qu'un pas vers le libéralisme a été franchi. Nous avons vu que certains inventeurs de l'ANB cherchaient, en fait, une libéralisation qui allait dans ce sens.

C'est au Nouveau-Brunswick que la demande du Secrétaire d'État aux colonies reçoit la réponse la plus favorable. Au début du mois de mai 1853 , les tarifs sont désormais d'un peu plus de cinq livres pour les sujets britanniques et de 50 , pour les autres ${ }^{24}$. Cependant cette loi, en défavorisant les inventeurs américains, projetait les relations canado-américaines à l'avant-scène.

\section{LES CRITIQUES AMÉRICAINES}

William McDougall, qui fit une enquête aux États-Unis au cours de 1853, soulignait dans son rapport au ministre de l'Agriculture l'insatisfaction des inventeurs américains quant à la loi des brevets de la Province du Canada ${ }^{25}$. Bien qu'auparavant d'opinion contraire, il est maintenant convaincu que des changements à la loi des brevets s'imposent afin que les inventeurs étrangers puissent obtenir des brevets de la Province. Il soutient que la loi actuelle place les inventeurs américains dans une situation difficile et mentionne que les États-Unis utilisent, comme mesure de réprésailles, l'adoption d'une loi dans l'État de New York interdisant la «contrebande» de marchandises canadiennes issues du piratage d'inventions américaines ${ }^{26}$. De plus, selon McDougall, le but de la loi en vigueur dans la province permettre l'introduction rapide de la technologie américaine - n'a pas été atteint autrement que dans des circonstances fortuites ${ }^{27}$. McDougall poursuit en disant que les lois protectionnistes en matière de brevets ne sont pas conséquentes avec le désir de réciprocité en matière commerciale. Or 1854 est l'année de la signature du Traité de

23. 16 Vict. c. 21 .

24. 16 Vict. c. 32 .

25. Ils étaient probablement insatisfaits de toutes les lois de l'ANB. Depuis la création du Bureau d'Agriculture en 1852, bureau qui deviendra ministère plus tard, c'est le ministre de l'Agriculture qui administre le système de brevets dans la Province du Canada.

26. La situation d'infériorité proviendrait du fait que les prix des marchandises fabriquées au Canada à partir de brevets américains seraient moins élevés que les prix des mêmes marchandises fabriquées aux États-Unis. La différence provenant du fait qu'au Canada il n'y ait pas de coûts associés à l'utilisation de brevets américains.

27. La raison évoquée à cet effet est la suivante: les dépenses pour copier une invention étant élevées, personne n'ose le faire de peur de voir les avantages qui en découleraient s'évanouir par l'introduction généralisée de la dite invention dans l'industrie en question. 
réciprocité canado-américain. McDougall propose finalement que les étrangers puissent obtenir des brevets au Canada-Uni à la condition qu'ils y manufacturent ou vendent l'invention brevetée ${ }^{28}$.

Les vœux de McDougall ne sont pas totalement ignorés, car trois projets de loi seront présentés à l'Assemblée législative entre 1852 et 1861: deux par John S. Sanborn, député de Sherbrooke, le premier en septembre 1854 et le deuxième en mars 1856 ; le troisième est présenté par François-Xavier Lemieux en février 1859. Les trois projets, qui prévoient la possibilité pour les inventeurs étrangers d'obtenir des brevets de la Province, seront abandonnés ${ }^{29}$.

Les partisans du projet de 1854 espéraient que son adoption favoriserait l'introduction de capital étranger dans la Province par l'établissement de nouvelles entreprises. Certains députés appuyèrent ce projet, parce que les entrepreneurs avaient du mal à trouver du capital dans la province à tel point qu'il leur était difficile d'investir dans des inventions, mêmes américaines et même si ces dernières pouvaient être copiées sans contraintes ${ }^{30}$.

George Étienne Cartier s'opposa au projet de Sanborn. Il soutint que s'il était adopté les inventeurs américains auraient de sérieux avantages sur les inventeurs de la Province, puisqu'ils pourraient faire breveter leurs inventions tant aux États-Unis que dans la Province alors que les inventeurs canadiens ne le pourraient pas à cause des tarifs trop élevés aux États-Unis (selon la loi adoptée en 1836). Cartier ne voyait pas pourquoi la Province se départirait de l'avantage de pouvoir profiter des inventions américaines à peu de frais ${ }^{31}$.

Le président du Conseil exécutif et premier ministre, Alan $\mathrm{N}$. $\mathrm{MacNab}$, et l'inspecteur général, William Caylay, ont appuyé Cartier de même que Jacob De Witt, homme d'affaires bien connu du BasCanada $^{32}$. John A. Macdonald, alors procureur général, réfuta l'idée

28. «Documents Submitted by the Bureau of Agriculture to the Legislature of Canada», JLAPC (1854), appendice II.

29. DLAUC (1854-1855): 233, 340, 476 et 693-696 et (1856): 981; Parliamentary Debates (PD) (s.1., Canadian Library Association, s.d.), 28 février 1856 et 31 mars 1856; JLAPC (1856), 143 et (1859), 67, 102, 503 et 558; et «Proceedings of the Board of Arts and Manufactures of Upper Canada», JBAMUC, 1 (1861): 43. Il se peut que le projet de 1856 ait été abandonné tout simplement parce que le gouverneur général ordonnait à la même époque un changement de gouvernement. William Hamilton Merritt, homme d'affaires fort connu et un des principaux architectes du Traité de réciprocité, présenta en 1856 un projet de loi spécial en faveur d'un inventeur américain au moment où Sanborn présentait le sien.

30. DLAUC (1854-1855), 233, 340, 476 et 693-696.

31. Idem.

32. Il faut souligner que «en 1849, [De Witt] occupe le poste de vice-président de l'Association for the Encouragement of Home Manufactures. (...) D'autre part, il préconise en 1849 et 1850 des mesures protectionnistes pour stimuler les manufactures locales». Voir Jean-Claude Robert, Dictionnaire biographique du Canada, 8: 243-245. 
selon laquelle ce projet favoriserait l'introduction de capital au pays. Selon Macdonald, les inventeurs étrangers, surtout américains, auraient eu plutôt tendance à vouloir vendre leurs brevets ou à vendre plus cher leurs produits brevetés dans la Province. Pour Macdonald, accorder des brevets aux Américains aurait signifié à leur donner des moyens de contrôler le développement industriel. Le solliciteur général Dunbar Ross appuya l'affirmation de Macdonald en disant que si les Américains ont tant de capital à leur disposition, ils devraient s'associer à des manufacturiers de la Province qui, eux, pourraient prendre des brevets pour exploiter les inventions américaines ${ }^{33}$.

Le projet de Lemieux était plus nuancé que ceux de Sanborn. Il prévoyait accorder aux inventeurs étrangers le droit d'obtenir des brevets de la Province du Canada selon le principe de réciprocité, c'est-à-dire que les étrangers auraient les mêmes tarifs à payer que les inventeurs de la Province en auraient à payer dans les pays étrangers ${ }^{34}$. Cette formulation reflète une volonté libérale en matière de brevets, tout en tenant compte du protectionnisme américain.

Cartier, devenu procureur général, s'y serait encore opposé avec force. Il aurait soutenu qu'il ne serait pas réaliste de placer la situation des inventeurs de la Province à égalité avec celle des inventeurs américains, ces derniers étant supportés par une économie beaucoup plus forte $^{35}$.

La prépondérance du protectionnisme en matière de brevets amenait par conséquent certains inventeurs à faire des demandes de lois spéciales. Ces demandes furent assez fréquentes ${ }^{36}$, ce qui ne pouvait que rendre la question des lois des brevets plus présente. D'ailleurs, en février 1861, à la suite de nombreuses demandes spéciales adressées à la législature de la Nouvelle-Écosse, un comité spécial chargé d'étudier la possibilité de libéraliser la loi des brevets est mis sur pied et un projet de loi, déposé. Mais ce projet de loi subira le

33. DLAUC (1854-1855), 233, 340, 476 et 693-696.

34. «Proceedings of the Board of Arts and Manufactures of Upper Canada», JBAMUC, 1 (1861): 43.

35. Scientific American (SA), 14,40 (11 juin 1859): 334. Notons immédiatement que la revue Scientific American était à l'époque la revue par excellence des inventeurs et des demandeurs ou détenteurs de brevets. Durant les années 1850, elle porta un sous-titre très évocateur: «The Advocate of Industry, and Journal of Scientific, Mechanical and Other Improvements».

36. Il est difficile de dire si ces demandes spéciales s'inscrivent dans un courant de pensée particulier, les informations recueillies n'étant pas assez explicites. Il y eut une seule demande spéciale en Nouvelle-Écosse en 1853, 2 en 1854, 4 en 1857, 4 en 1859 et 6 en 1860; au Canada-Uni, c'est 1 en 1853, 3 en 1855, 2 en 1856, 1 en 1857, 2 en 1858, 2 en 1859 et 1 en 1860. Pour le Nouveau-Brunswick, c'est 2 en 1853 et 1 en 1855 . Dans ce dernier cas, il faut rappeler que seuls des tarifs plus élevés constituent une barrière pour les inventeurs étrangers. 
même sort que ceux présentés à la législature de la Province du Canada $^{37}$.

Le courant libéral ne s'exprimait pas seulement dans les différentes législatures canadiennes: des lettres sont envoyées au ministre de l'Agriculture de la Province du Canada ${ }^{38}$ ou publiées dans les journaux ${ }^{39}$; des pétitions parviennent à l'Assemblée législative du Nouveau-Brunswick ${ }^{40}$; des débats sur la question des lois des brevets se tiennent au sein de la Chambre des Arts et Manufactures du BasCanada $^{41}$. Le libéralisme est également présent au ministère de l'Agriculture de la Province du Canada ${ }^{42}$.

Les pressions en faveur de la libéralisation des lois sur les brevets de l'ANB viennent autant de l'extérieur, notamment des États-Unis, que de l'intérieur. En plus de la loi adoptée par l'État de New York dont William McDougall faisait état, en 1854, dans son rapport au ministre de l'Agriculture, la revue Scientific American fit paraître quelques articles dénonçant la loi britannique de 1852 ainsi que le protectionnisme canadien ${ }^{43}$. La revue s'objecte aux arguments de ceux qui, comme Cartier, soutiennent qu'un loi libérale favoriserait les inventeurs américains. Selon la revue américaine, ce sont les inventeurs canadiens qui profiteraient le plus de lois libérales, car la valeur des brevets américains est bien plus grande que celle des brevets cana-

37. JHANS, 13 et 21 février 1861,24 et 25 mars 1861 et 8 avril 1861 .

38. En février 1857, F. G. Willson, un inventeur du Haut-Canada ayant obtenu des brevets en 1850 et 1855 , envoie une lettre au ministre de l'Agriculture, P. M. Vankoughnet, dans laquelle il favorise la réciprocité en matière de brevets. ANC, RG17 AI2, vol. 1491, 694. Pour les brevets de Willson, voir List of Canadian Patents (...).

39. C. B. Thompson, dans le St.Catherines Journal, plaide en faveur d'une loi qui accorde aux étrangers les mêmes droits qu'aux inventeurs locaux. Thompson affirme que sa position est appuyée par William Hamilton Merritt. C. B. Thompson, "The Patent Laws», St.Catherines Journal, 5 mars 1856 (CIHM, $\mathrm{n}^{\circ}$ 63043).

40. Une première pétition présentée en février 1857 , signée par plus de 70 personnes, demande que les tarifs soient rendus égaux pour tous. Une deuxième pétition, signée par plus de 35 personnes et présentée le mois suivant, demande vraisemblablement la même chose. Journals of the House of Assembly of the Province of New Brunswick (JHAPNB) (1857), 23 et 60 .

41. ANC, RG17 AI3, vol. 1627, 4 janvier 1858. Certains membres des Chambres des Arts et Manufactures sont ou seront des détenteurs de brevets; voir Denis Veilleux, op. cit., 70, note 38 .

42. Dans une réponse à J. P. Leprohon, secrétaire d'un comité spécial chargé d'enquêter sur la loi des brevets de la province, William Hutton, secrétaire du ministre de l'Agriculture, favorise une loi libérale. Ce dernier pense que si les étrangers pouvaient obtenir des brevets dans la Province du Canada, le commerce serait grandement stimulé. Il faudrait cependant, selon lui, que les étrangers soient encouragés à établir des entreprises dans la Province. Un tel libéralisme, soutient Hutton, permettrait la venue de capital étranger au pays. Voir ANC, RG17 AI2, vol. $1492,390$.

43. SA, 8,48 (13 août 1853): $381 ; 8,51$ (3 septembre 1853): 405; 14,2 (16 avril 1859): 270 ; 14,40 (11 juin 1859): 334 ; et 2,6 (4 février 1860): $89-90$, deuxième série. 
diens $^{44}$. Mais Scientific American concède que la loi américaine devrait être changée pour que cela soit possible. Elle admet d'ailleurs que le protectionnisme américain est un frein réel à l'adoption, par les provinces de l'ANB, de lois sur les brevets à caractère libéral ${ }^{45}$.

Plusieurs rapports des commissaires américains des brevets appuient les recommandations de Scientific American à l'effet que des changements de lois seraient souhaitables des deux cotés de la frontière canado-américaine ${ }^{46}$. Dans son rapport annuel de 1855 , le commissaire soulignait, à sa manière, les enjeux commerciaux derrière les débats opposant protectionnistes et libéraux:

\begin{abstract}
All machines invented here, can be made and used in Canada without any license from the American patentee; and the products of those machines can, with little trouble or expense, be brought into our markets to compete with like commodities manufactured here by persons who are obliged to pay for the right to use the patented machines for that purpose. This operates like a discriminating tariff against the home manufacturer, which cannot but be prejudicial to his interests ${ }^{47}$.
\end{abstract}

Il ajoute que les États-Unis devraient prendre les devants, c'est-àdire adopter des mesures libérales et plus précisément rendre les tarifs égaux pour tous. Cela ne pourrait, selon lui, qu'encourager les provinces de l'ANB à s'engager à leur tour vers le libéralisme et redonner aux inventeurs américains les avantages que les brevets confèrent ${ }^{48}$. Son argumentation sera reprise par Scientific American ${ }^{49}$ et le St.Catherines Journal ${ }^{50}$ au Haut-Canada.

$\mathrm{Si}$, en 1861 , les partisans du libéralisme en matière de brevets n'ont pas encore obtenu les résultats escomptés au Canada, il en va tout autrement aux États-Unis.

\title{
LA LIBÉRALISATION DE LA LOI AMÉRICAINE SUR LES BREVETS D'INVENTIONS ET LA RÉSISTANCE DE LA PROVINCE DU CANADA
}

Le 2 mars 1861, les États-Unis amendent leur loi des brevets d'une manière significative. L'article 10 stipule que les tarifs

44. Ibid., 14,40 (11 juin 1859): 334 .

45. Ibid., 8,51 (3 septembre 1853): 405, et 2,6 (4 février 1860): 89-90, deuxième série.

46. Report of the Commissioner of Patents (...) (Washington, 1854): 16-18, 1 (1855): 5 , et 1 (1858): 12-13.

47. Ibid., 1 (1855): 5. Le commissaire évoque en fait les raisons pour lesquelles la loi de l'État de New York contre la «contrebande», citée par William McDougall, a été adoptée.

48. Idem., et ibid., 1 (1858): 12-13.

49. $S A, 2,6$ (4 février 1860): 89-90, deuxième série.

50. C. B. Thompson, loc. cit. 
discriminatoires imposés aux inventeurs étrangers sont réaménagés selon un principe de réciprocité, c'est-à-dire que les tarifs pour les inventeurs étrangers habitant dans des pays qui n'exercent pas de discrimination envers les inventeurs américains seront les mêmes, aux États-Unis, que pour les inventeurs américains ${ }^{51}$. L'adoption de cette loi allait raviver les tensions entre protectionnistes et libéraux au Canada, particulièrement dans la Province du Canada.

Dans les Maritimes, on a mis peu de temps à réagir à la nouvelle loi américaine. En Nouvelle-Écosse, au moment où l'Assemblée législative doit composer avec huit demandes spéciales, le procureur général, A. G. Archibald, présente un projet de loi, en mars 1862, qui prendra effet en avrili ${ }^{52}$. Le droit d'obtenir des brevets de la NouvelleÉcosse est maintenant accordé aux personnes résidant depuis au moins un an dans la Province au moment de la demande ${ }^{53}$. Les étrangers peuvent donc, s'ils remplissent cette condition, y obtenir des brevets. Un petit pas est fait en faveur des Américains, mais c'est un recul pour les inventeurs des autres provinces de l'ANB. Au NouveauBrunswick, dès avril 1862, une nouvelle loi est également adoptée. Les tarifs discriminatoires pour les étrangers sont remplacés par des tarifs selon le principe de réciprocité ${ }^{54}$. Ici, c'est un gain réel pour les partisans du libéralisme ainsi que pour les inventeurs.

Trois ans plus tard, en Nouvelle-Écosse, la libéralisation fera un nouveau pas. Le 28 février, deux pétitions sont présentées à l'Assemblée législative par A. G. Archibald: des inventeurs américains demandent des lois spéciales afin qu'il leur soit permis d'obtenir des brevets pour des inventions concernant l'extraction de l'or dans le minerai. Lors du débat entourant ces pétitions, Samuel L. Shannon dit appuyer tout projet de loi sur les brevets qui va dans le sens de la réciprocité, tandis que $\mathrm{A}$. McFarlane soutient que la loi actuelle est nuisible à la Province puisque de larges quantités de minerai sont envoyées aux États-Unis pour y être traitées afin d'obtenir de l'or ${ }^{55}$.

Une semaine plus tard, Shannon présente un projet de loi à l'Assemblée législative. Celui-ci est basé sur le principe de réciprocité car ce serait un bon moyen, dit Shannon, de faciliter le développement de l'industrie ${ }^{56}$. Archibald préférerait plutôt que l'on privilégie les

51. Act of 1861 , chapitre 88 .

52. JHANS (1862), 46, 49, 69, 70 et 101.

53. 25 Vict. c. 27.

54. 25 Vict. c. 33.

55. Debates and Proceedings of the House of Assembly of the Province of Nova Scotia (DPHAPNS), 28 février 1865, 37.

56. JHANS (1865), 41, 42, 104, 106 et 111. 
inventions que l'on ne pourrait pas s'approprier à cause du secret industriel. Isaac LeVesconte craint que le projet de Shannon ne laisse la porte ouverte à l'envahissement des Américains ${ }^{57}$. Malgré qu'Archibald ait présenté de nouvelles pétitions provenant de mineurs qui réclament qu'il soit plus facile d'obtenir des brevets de la NouvelleÉcosse, le projet ne sera pas adopté par l'Assemblée législative ${ }^{58}$.

Mais le 16 avril, Archibald revient à la charge et présente un nouveau projet de loi qui, lui, sera accepté ${ }^{59}$. La nouvelle loi de la Nouvelle-Écosse épouse d'ailleurs les opinions de Archibald. Les inventeurs étrangers, c'est-à-dire ceux qui ne sont pas résidents de la Nouvelle-Écosse depuis au moins un an, peuvent désormais obtenir des brevets, mais uniquement pour des inventions ou découvertes fondées sur les principes de la chimie ou des secrets scientifiques en relation avec l'amalgamation, la séparation ou la production d'or à partir de quartz ou autres substances. Ces inventions ne doivent cependant ni être connues ni être en usage dans la Province ${ }^{60}$. Comme le développement de l'industrie de l'exploitation du minerai aurifère est intense à l'époque, l'aspect libéral de cette loi peut être associé à une volonté de développement économique. C'était là un argument fondamental des partisans du libéralisme.

Alors que, chacune à leur manière, les provinces maritimes se sont engagées dans une voie libérale en matière de brevets, la Province du Canada reste sur ses positions protectionnistes, malgré les pressions considérables en faveur du libéralisme: des projets de loi publique sont présentés en $1861(1)^{61}, 1862(1)^{62}, 1863(1)^{63}$ et $1865(2)^{64}$; de nombreuses demandes de lois spéciales faites par des inventeurs étrangers ou en leur nom parviennent aux différentes législatures entre 1861 et $1867^{65}$; des pétitions et des projets de loi en faveur de la libéralisation sont formulés par les Chambres des Arts et Manu-

57. DPHAPNS, 7 mars $1865,70-71$.

58. JHANS (1865), 52.

59. Ibid. (1865), 116, 117, 118, 122, 123 et 128 .

60. 28 Vict. c. 4.

61. JLAPC (1861), 118 et 361, et Dunbar Browne, loc. cit., 291.

62. JLCPC (1862), 101, 125 et 158, et JBAMUC, 2 (1862): 152-153.

63. "Annual Report of the Board of Arts and Manufactures of Upper Canada», JBAMUC, 4 (1864): 7. Le projet est vraisemblablement abandonné parce que des élections sont prévues.

$64 . J L A P C, 27$ et 30 janvier 1865,8 et 9 février 1865.

65. Trois de ces demandes spéciales sont faites en Nouvelle-Écosse en 1861 et sept, en 1862. Pour la Province du Canada, c'est 2 en 1861, 1 en 1863, 1 en 1864, 6 en 1866 et 1 en 1867. La loi de 1861 du Nouveau-Brunswick éliminait pratiquement la nécessité de telles demandes. Il faut noter que d'autres demandes spéciales visaient à octroyer à des Canadiens des brevets sur des inventions faites par des étrangers, notamment américains. Les législatures pouvaient donc percevoir des signaux contradictoires. Ces informations ont été tirées des Journaux et des Débats. 
factures du Haut- et du Bas-Canada ${ }^{66}$ et par l'Institut des Artisans de Hamilton et Gore ${ }^{67}$; et finalement, des articles de journaux sont publiés ${ }^{68}$. Les rapports annuels du ministre de l'Agriculture sont également favorables au libéralisme ${ }^{69}$.

Mais à partir de 1864 , tout en demeurant favorable à la libéralisation de la loi, le ministre pense que la probabilité d'une fédération des colonies de l'ANB amènera une révision des politiques ${ }^{70}$. Il n'est pas le seul à penser ainsi. Lors de discussions à propos de huit demandes spéciales adressées à l'Assemblée législative au cours de 1866, Cartier, qui défendait depuis plusieurs années des positions protectionnistes, soutient qu'une fois la Confédération créée, il pourrait bien favoriser une attitude plus libérale en matière de brevets. En attendant, il croit cependant qu'il vaut mieux s'en tenir à la politique protectionniste, d'autant plus qu'une attitude plus libérale pourrait mettre quelques manufacturiers dans des positions difficiles, puisqu'ils utilisent déjà des inventions étrangères ${ }^{71}$.

La Chambre des Arts et Manufactures du Haut-Canada fit justement remarquer à la même époque que si la Confédération venait à voir le jour, la valeur des brevets canadiens serait alors plus grande, ce qui favoriserait les inventeurs canadiens ${ }^{72}$. Les intérêts des inventeurs canadiens deviennent un argument de plus en plus utilisé pour promouvoir le libéralisme en matière de brevets. Pour plusieurs de ses promoteurs, celui-ci permettrait aux inventeurs canadiens de bénéficier des dispositions de la loi américaine de 1861, basée sur le principe

66. Des pétitions sont envoyées à la législature de la Province du Canada en 1861, 1862, $1863,1864,1865,1866$ et un projet de loi en 1863. Certains rapports annuels des Chambres au ministre de l'Agriculture favorisent également une libéralisation tout comme des articles du Journal of the Board of Arts and Manufactures for Upper Canada. Voir ANQ, 06M-P82.2, Chambre des Arts et Manufactures du Bas-Canada, Correspondance 1857-1873, 9 mars 1861, 20 janvier 1863, 7 février 1863, 13 mars 1863, 25 mars 1863 et 4 avril 1863, JLAPC (1861), 27; (1862), 66 et 176; (1863), 44 et 70; (1864), 24 et 128; (1865), 89; JBAMUC, 1 (1861): 43; 1862), 133-135 et 152-153; 4 (1864): 65-68, 73-74 et 204-205; 7 (1867): 32; JLCPC (1863), 64; Chambre des Arts et Manufactures du Bas-Canada, «Annual Report», Sessionnal Papers (SP), 6,6 (1865); idem., 3 (1867): 64; et ANC, RG17 AIl, vol. 3, dossier 98.

67. JLAPC (1864), 192.

68. Scientific American cite en novembre 1861 un article de The Progressionist, publié à Morpeth au Haut-Canada, qui défend le droit des inventeurs étrangers de prendre des brevets canadiens. Si un tel droit était respecté, l'économie se développerait plus rapidement y soutienton. Voir $S A, 5,18$ (2 novembre 1861): 284.

69. «Annual Report of the Minister of Agriculture and Statistics (...)», SP, 4 (1863): ix; idem., 32 (1864): ix; et idem., 3 (1867): ix.

70. Idem., 5 (1866): 15, et idem., 3 (1867): ix.

71. Canada Parliamentary Debates (CPD) (s.1., Canadian Library Association, s. d.), 15, 25 et 26 juin 1866,9 et 31 juillet 1866 .

72. «Annual Report», JBAMUC, 5 (1865): 33-36, et idem., 6 (1866): 35. 
de réciprocité ${ }^{73}$. De plus, le Journal of the Board of Arts and Manufactures for Upper Canada soutient que, tout comme les inventeurs, le public entier bénéficierait d'une libéralisation de la loi des brevets ${ }^{74}$. Le ministre de l'Agriculture souligne ce que pourraient être ces bénéfices sur le plan économique: l'introduction de nouvelles industries dans la Province et la création de plusieurs emplois, notamment. Cela apparait d'autant plus évident au ministre que les pays s'étant engagés dans cette voie ne s'en sont jamais plaints ${ }^{75}$. La plupart des partisans du libéralisme partagent toujours cet avis, dont à plus forte raison Scientific American ${ }^{76}$.

Outre l'avènement probable de la Confédération comme facteur retardant la libéralisation de la loi sur les brevets de la Province du Canada, il faut également mentionner les négociations en vue du renouvellement du Traité de réciprocité. Dès 1865, le ministre de l'Agriculture soutient que la libéralisation de la loi des brevets est intimement liée à ce renouvellement ${ }^{77}$.

Scientific American rétorque que le renouvellement du Traité de réciprocité ne devrait se faire que s'il englobe la question des brevets, et que le Canada doit maintenant faire des concessions ${ }^{78}$. Plus tard, la revue soulignera que les Américains ont fait leur bout de chemin vers la réciprocité et déplorera que «there is one point on which Canadian Reciprocity is all on one side, like the handle of a jug: we refer to their patent system $^{79}$ ».

La loi du Nouveau-Brunswick adoptée en 1861 ne fait pas l'objet de telles réprobations. Au contraire, un article de Scientific American en fait l'éloge: «The people of New Brunswick are among the most vigorous, enterprising and intelligent in all British Colonies.» L'article ne manque pas de faire remarquer que c'est une bonne loi du point de vue des inventeurs américains ${ }^{80}$.

Si l'anticipation de la Confédération a probablement retardé l'adoption d'une loi libérale sur les brevets dans la Province du Canada, et aussi en Nouvelle-Écosse, l'avènement de la Confédération a par contre sans doute créé une condition favorable à cette adoption.

73. «Proceedings of the Sub-Committee», JBAMUC (1862): 133-134, et «Annual Report of the Minister of Agriculture and Statistics (...)», SP, 32 (1864).

74. JBAMUC, 4 (1864): 65-68 et 204-205.

75. «Annual Report of the Minister of Agriculture (...)», SP, 32 (1864).

76. $S A, 6,22$ (31 mai 1862): 345 .

77. «Annual Report of the Minister of Agriculture (...)», SP (1865), 11-12, ANC, RG17

AI1, vol. 4, dossier 143 et ANC, RG17 AI2, vol. 1492, 52.

78. $S A, 14,6$ (3 février 1866): 87.

79. Ibid., 10,6 (6 février 1864): 86 , et 10,8 (20 février 1864): 8 .

80. Ibid., 6,22 (31 mai 1862): 346 . 
Mais tant que le renouvellement du Traité de réciprocité était perçu comme possible ou peu éloigné dans le temps, cette condition n'était pas suffisante.

\section{LA CONFÉDÉRATION ET LA QUESTION DES BREVETS}

Les rangs des partisans du libéralisme grossissent au début de 1867, quelques mois avant l'avènement de la Confédération. À leurs récriminations s'ajoutent celles de Charles Legge, de l'agence de brevets Charles Legge and Company, qui entreprend au début de 1867 une campagne en faveur d'une loi libérale. Les intérêts de Legge sont assez évidents, puisqu'une telle loi lui amènerait de nombreux clients, américains notamment.

Une brochure de la compagnie, vraisemblablement publiée au début de 1867, souligne que les lois de la Province du Canada, de l' ̂̂le-du-Prince-Édouard et de la Nouvelle-Écosse se rangent aux côtés de celles de la Suisse, de la Turquie, de la Chine et du Japon quant à leur non-libéralité, et que ces provinces ne devraient pas être fières d'être ainsi comparées. Elles devraient plutôt se placer parmi les «enlightened progressive nations» en adoptant des lois libérales. L'auteur affirme que de telles lois stimulent le développement industriel. Les exemples donnés sont, notamment, les États-Unis, l'Angleterre et la France. La brochure souligne également que les inventeurs du Canada voient un marché énorme leur échapper, les ÉtatsUnis, car ils doivent payer très cher les brevets américains (500\$). Le document propose quand même une restriction: les inventions d'étrangers doivent être disponibles à un prix raisonnable dans les 18 mois suivant l'octroi ${ }^{81}$. Le Journal of the Board of Arts and Manufactures for Upper Canada et Scientific American feront référence, plus tard, à la brochure de la Charles Legge and Company ${ }^{82}$.

La naissance de la Confédération avantagea certainement les partisans du libéralisme en matière de brevets: le gouvernement canadien ne pouvait pas faire autrement que d'adopter une loi unique pour l'ensemble des provinces, une loi qui concilierait le libéralisme prévalant dans les provinces atlantiques et le protectionnisme québécois et ontarien. Sans une telle loi, la nouvelle Confédération engendrerait des tensions dangereuses, comme le soulignera un article du Evening Telegraph de Montréal, rapporté dans Scientific American ${ }^{83}$. Devant

81. Charles Legge \& Co., Suggestions with Reference to the Proposed New Act Respecting Letters Patent for Inventions in the Dominion of Canada (Montréal, 1867).

82. JBAMUC, 8 (janvier 1868): 1-3.

83. $S A, 18,22$ (30 mai 1868): 346 . 
l'adoption inévitable d'une loi valide pour l'ensemble des provinces, les deux camps chercheront à exercer leur influence.

Pendant que la Chambre des Arts et Manufactures du Bas-Canada fait parvenir au Sénat canadien une pétition demandant la libéralisation de la loi des brevets ${ }^{84}$, Legge poursuit sa campagne en envoyant une lettre au secrétaire d'État, qui la refile au ministre de l'Agriculture. Legge y affirme que la première loi de la Confédération en matière de brevets devrait être libérale, à défaut de quoi il craint que les Américains n'éliminent totalement la possibilité pour les inventeurs canadiens de breveter aux États-Unis ${ }^{85}$. Un inventeur, Richard Lewis, détenteur de plusieurs brevets, poursuit dans la même veine. Il affirme, dans une lettre au ministre de l'Agriculture, qu'une loi libérale en matière de brevets serait profitable aux inventeurs canadiens. Une telle loi, dit Lewis, ouvrirait un marché de 34 millions de personnes aux inventeurs canadiens. Il souligne ainsi la grande valeur des brevets américains ${ }^{86}$.

Le gouvernement canadien reçoit ainsi des opinions contraires: les partisans du protectionnisme se manifestent eux aussi. John McBean, détenteur d'un brevet de la Province du Canada, réplique à la Charles Legge and Company, dans une brochure parue en 1867, que les lois européennes et américaines accordent des brevets au premier demandeur (et non nécessairement au véritable inventeur). Il ne voit pas alors pourquoi un Canadien ne pourrait pas prendre un brevet sur une invention étrangère. Il rejette donc le libéralisme. Et cela d'autant plus qu'il contredit l'affirmation selon laquelle le libéralisme en matière de brevets profiterait à l'économie canadienne. McBean soutient que le Canada serait plutôt envahi de brevets détenus par des Américains. Conséquemment, il demande que les Canadiens puissent breveter aux États-Unis les «découvertes» de nouvelles inventions qu'ils font comme ils peuvent le faire pour les inventions européennes ${ }^{87}$. De cette façon, les inventeurs canadiens ne seraient plus tentés d'émigrer aux États-Unis et feraient ainsi profiter leur pays de leur inventivité88.

84. Journals of the Senate (JS) (1868), 84.

85. ANC, RG17 AI7, vol. 1664, dossier 734 .

86. ANC, RG17 AI7, vol. 1164, dossier 128. Pour les brevets de Lewis, voir List of Canadian Patents (...). Il en a obtenus 5 entre 1862 et 1868.

87. Depuis 1849, dans la Province du Canada, devenue le Québec et l'Ontario, il était possible pour ses résidents et sujets britanniques de breveter des inventions européennes «découvertes» faites lors de voyages, par exemple. Cela n'était pas le cas pour les «découvertes» faites aux États-Unis, car on cherchait à ce que les inventions américaines aient dans la Province le plus large accès possible. La loi de 1849 de la Province du Canada avait emprunté à cet égard, à la loi bas-canadienne de 1831 .

88. McBean, John, The Patent Laws (Ottawa, s.é., 1867). Pour les brevets de McBean, voir List of Canadan Patents (...). 
Le ministre de l'Agriculture, quant à lui, a nettement changé de camp. Il soutient que «the peculiar circumstances of this country, however be pleaded in support of this provision of Canadian law», c'est-à-dire qu'il soutient l'exclusion des inventeurs étrangers ${ }^{89}$.

Plusieurs pétitions nous montrent aussi les avis contradictoires reçus par la législature canadienne. D'une part, elle reçoit treize pétitions qui demandent que les Américains, surtout, ne puissent pas breveter au Canada; que les inventions américaines puissent être brevetées par les inventeurs locaux, que les brevets émis au NouveauBrunswick ne s'étendent pas à l'ensemble du Canada afin que les brevets qui y sont détenus par les Américains ne s'appliquent pas au reste du pays ${ }^{90}$. Ces pétitions sont donc une manifestation du protectionnisme le plus radical ${ }^{91}$. D' autre part, la Chambre des communes et le Sénat reçoivent une pétition de Prowse Bros. et autres en faveur d'une loi libérale, c'est-à-dire permettant aux étrangers de breveter au Canada afin que les inventeurs canadiens puissent breveter à l'étranger, particulièrement aux États-Unis ${ }^{92}$.

C'est dans cette situation de tensions manifestement vives que le 5 mai 1868 J. C. Chapais, ministre de l'Agriculture du Canada, présente un projet de loi au Sénat. Le projet prévoit que les étrangers pourront obtenir des brevets canadiens après avoir résidé un an au Canada. De plus, les brevets déjà accordés dans les provinces de la Confédération ne s'étendront pas à l'ensemble du Canada. Les débats eurent lieu du 11 au 13 mai et opposèrent surtout Sanborn et, curieusement, Peter Mitchell, député du Nouveau-Brunswick et partisan du protectionnisme ${ }^{93}$.

89. Le ministre fait référence à la loi de l'ancienne Province du Canada, devenue le Québec et l'Ontario, toujours en vigueur. Voir «Report of the Minister of Agriculture of the Province of Canada for the First Six Months of the Year 1867, Preceeding the Confederation», SP (1869): 8-9.

90. La crainte que les Américains n'envahissent le Canada avec leurs inventions peut être soutenue par le nombre de brevets obtenus par des Américains au Nouveau-Brunswick. Pour l'année 1867, sur les 41 brevets, dont le lieu de résidence de l'inventeur est connu, 28 sont détenus par des Américains, soit 68\%. Voir The Year Book and Almanac of Canada (Montréal, John Lowe and Company of Montreal, 1868), 107.

91. Plus de 750 signatures sont apposées au bas de ces pétitions. Un peu plus de $30 \%$ des signataires ayant indiqué une profession $(83 \%)$ sont liés au secteur industriel ou aux transports et $30 \%$, au secteur du commerce. ANC, RG17 AI1, vol. 19, dossiers 1647, 1648, 1653, 1654, $1665,1667,1668,1677,1682,1683$ et 1684 et vol. 20, dossier 1724; Journals of the House of Commons (JHC) (1867-1868), 143,148,155,164, 168, 170,176,179, 183, 206 et 224, et $J S$ (1867-1868), 84, 183, 200, 202, 207, 214, 216, 227, 243, 250, 277.

92. George R. Prowse et George F. Prowse recevront 5 brevets entre 1854 et 1868; voir List of Canadian Patents (...). Un peu plus de soixante signatures apparaissent sur cette pétition. Seulement sept signataires ont indiqué leur profession et elles sont toutes liées au secteur manufacturier, voir ANC, RG17 AI1, vol. 20, dossier 1755 .

93. Debates of the Senate (DS) (1867-68), 246, 275-279, 287-288 et 290. 
Les opposants à la reconnaissance des droits des inventeurs étrangers soutiennent qu'étant donné la jeunesse du Canada et sa faible capacité de compétition en matière de brevets, il ne devrait pas être permis aux étrangers de prendre des brevets canadiens. Si cette situation était différente, disent-ils, et que les sentiments de la population allaient vers une volonté d'en finir avec des lois restrictives, on pourrait alors adopter un principe de réciprocité ${ }^{94}$.

Les partisans d'une loi libérale, comme Sanborn, soutiennent au contraire que les lois des brevets encouragent l'entreprise et développent l'économie, le cas des États-Unis étant alors invoqué. L'obligation de produire au pays les inventions brevetées est le moyen principal d'y parvenir. Les partisans font également référence aux lois étrangères qui sont libérales et au principe de réciprocité incorporé à la loi américaine ${ }^{95}$.

Les opposants rétorqueront à Sanborn, comme le fait Peter Mitchell, qu'ils ne sont pas d'accord pour faire le lien entre le développement économique et les lois des brevets. Ils soulignent que la croissance économique aux États-Unis s'est faite en dépit de la loi sur les brevets, et que la guerre civile a certainement aidé au développement économique autant, sinon plus, que cette loi. Les opposants soulignent également le cas du Nouveau-Brunswick où les étrangers peuvent prendre des brevets et où l'industrie ne se serait pas développée. Mitchell poursuit en disant que le libéralisme en matière de brevets n'est là que pour favoriser les inventeurs américains. Pour Mitchell, les Américains ne sont libéraux que lorsque cela fait leur affaire $^{96}$.

Malgré l'opposition au projet de loi, Sanborn propose que les brevets accordés jusqu'à maintenant dans les quatre provinces de la Confédération, et qui sont encore valides, puissent être étendus à tout le Canada. La proposition est adoptée à 21 voix contre 17 . Le lendemain de son adoption, Chapais annonce au Sénat que le gouvernement du Canada n'acheminera pas le projet au Sénat et qu'il est retiré de l'ordre du jour de la Chambre des communes ${ }^{97}$.

Mais entre le 18 et le 20 mai, un nouveau projet de loi est déposé. Il prévoit que les étrangers pourront obtenir des brevets canadiens s'ils ont résidé au moins un an au Canada avant la date de la demande. Il prévoit également que les Canadiens pourront obtenir des brevets sur des inventions étrangères (probablement dans les cas où l'inventeur

94. Idem.

95. Idem.

96. Idem.

97. Idem. 
étranger ne réclame pas de brevets au Canada). Le projet est adopté par la Chambre des communes, mais comme il n'est jamais devenu loi, on peut penser que c'est le Sénat qui, à son tour, l'a rejetég8.

Il est clair que l'un des camps devra céder. Ce n'est pas par hasard qu'en juin 1868, la Charles Legge and Company publie une nouvelle brochure. Celle-ci vise à attirer les inventeurs étrangers à prendre des brevets au Nouveau-Brunswick, grâce à ses bons offices bien entendu, dans l'espoir que plus tard ces brevets s'étendent à tout le Canada. Leggè cherche en fin de compte à créer un rapport de force en faveur d'une loi libérale. Plus il y aura d'étrangers détenteurs de brevets au Nouveau-Brunswick, moins le gouvernement du Canada ne pourra les ignorer dans une loi devant fatalement être adoptée pour l'ensemble de la Confédération ${ }^{99}$.

Au mois de mars 1869, Scientific American montre qu'elle comprend l'inévitabilité d'une loi canadienne sur les brevets plus ou moins libérale. Elle publie un article sur une proposition que leur avait fait parvenir Alexander Anderson, de London en Ontario, qui proposait aux inventeurs américains un moyen de contourner les lois canadiennes. La revue s'objecte à un tel procédé et conseille vivement à Anderson de travailler à libéraliser la loi ${ }^{100}$.

Dans ce contexte, le 26 avril 1869, Chapais présente un nouveau projet de loi devant le sénat canadien qui deviendra la première loi canadienne, après de longs débats dans les deux chambres de la législature. Le projet est relativement semblable à celui qu'avait présenté Chapais un an auparavant. Il prévoit que quiconque ayant résidé au Canada au moins un an avant une demande de brevet peut l'obtenir. Les brevets octroyés à des sujets britanniques qui résidaient dans une des provinces avant la Confédération peuvent demander que leurs brevets soient étendus à l'ensemble du Canada. Les Américains ayant obtenu des brevets du Nouveau-Brunswick depuis 1851 sont donc exclus. Finalement, le projet de loi prévoit que les Canadiens ne pourront breveter d'inventions étrangères ${ }^{101}$.

Sanborn n'est pas d'accord avec le projet tel que présenté et soutient que seule une loi libérale, c'est-à-dire sans critère de résidence

98. Debates of the House of Commons (DHC) (1867-68), 726-727, 746-747 et 751-752.

99. Charles Legge and Company, How and By Whom Letters Patent Can Be Secured for American, Foreign, and Other Inventors, in the Dominion of Canada [...] (Montréal, s.é., 1868).

100. SA, 20,13 (27 mars 1869): 201-202. Anderson a reçu quatorze brevets entre 1854 et 1868, la plupart valides dans la Province du Canada mais quelques-uns valides en NouvelleÉcosse ou au Nouveau-Brunswick; voir List of Canadian Patents (...), Lovell's Canadian Dictionary for 1871 (...) (Montréal, J. Lovell, [1871]), et The Year Book and Almanac of Canada, 1867-1868.

101. DS, 28 avril $1869,32-33$. 
obligatoire ou de nationalité, peut permettre un développement économique comme ce fut le cas aux États-Unis. Pour Sanborn, il faut cependant exiger que les inventions brevetées soient obligatoirement manufacturées au Canada, ce que n'exige pas le projet de loi de Chapais ${ }^{102}$.

Certains partisans du projet, comme John Ross et Jonathan McCully, sont aussi des opposants au système de brevets en général, car ils ne voient pas les brevets comme des instruments du bien-être public mais plutôt comme des monopoles. Ils redoutent par-dessus tout l'invasion possible des inventeurs américains au Canada. Ils voient donc d'un bon œil que les brevets du Nouveau-Brunswick détenus par des Américains ne puissent s'étendre à tout le Canada. Ils appuient le projet de loi de Chapais malgré tout, parce qu'il n'est pas trop libéral. Alexander Campbell, ministre des Postes, adopte une position semblable à celle de Ross et McCully. Mais il ajoute en plus que l'adoption d'une loi libérale en ce moment affaiblirait en quelque sorte la position du Canada dans les négociations pour le renouvellement du Traité de réciprocité ${ }^{103}$.

Chapais, dans la défense de son projet, avoue ne pas comprendre les objections de Sanborn. Il soutient qu'il est évident que le Canada doit protéger raisonnablement ses «industries naissantes contre leurs rivales plus anciennes, plus riches et plus favorisées» en parlant des États-Unis. Quant à Letellier de Saint-Just, qui appuie Sanborn, il ne comprend pas la logique de l'argumentation de Campbell. Il ne voit pas comment le projet de loi peut avoir une quelconque influence sur les négociations canado-américaines pour le renouvellement du Traité de réciprocité. Sanborn ajoute à cela que seule une loi libérale pourrait favoriser l'ingéniosité canadienne en ouvrant le marché américain aux inventeurs canadiens ${ }^{104}$.

Finalement, un amendement proposé par Sanborn, à l'effet de permettre à quiconque d'obtenir des brevets canadiens, est rejeté à 33 voix contre 12 . Le 3 mai, le Sénat adoptait le projet de loi de Chapais. Il devait maintenant recevoir l'assentiment de la Chambre des communes ${ }^{105}$.

Les débats commencent le 29 mai. A. T. Galt suggère une clause qui permettrait à n'importe quel inventeur d'introduire une invention au Canada à condition qu'elle soit fabriquée et employée au Canada dans les douze mois. Les opinions sont partagées à savoir si un tel

102. Idem.

103. Idem.

104. Ibid. (1869), 42-56, 60-68, 75-76 et 80 .

105. Idem. 
amendement serait favorable ou non aux Américains. Certains disent, comme Cartier et Macdonald, que les Américains auront tendance à prendre des brevets et à fabriquer l'invention au Canada sur une base régulière, mais à une échelle si réduite que les prix en seront exhorbitants. Les enjeux commerciaux sont ainsi évoqués une fois de plus. Tilley poursuit en disant que la loi du Nouveau-Brunswick a nui aux intérêts de ses habitants, car elle a fait en sorte de hausser les prix des produits sur lesquels une redevance aux inventeurs étrangers était ajoutée. Il est par conséquent contre une loi libérale ${ }^{106}$. McDougall, lui, montre combien les Américains accordent une priorité à la libéralisation des lois canadiennes ${ }^{107}$.

Pendant que la Chambre des communes discute du projet de loi de Chapais, de nouvelles pétitions lui parviennent ainsi qu'au Sénat. Cette fois, toutes les pétitions réclament l'élimination de tout critère de nationalité ou de résidence et une clause obligeant l'établissement et l'opération continuelle de manufactures qui fabriqueraient les inventions brevetées ${ }^{108}$.

$\mathrm{Si}$, comme en mai 1868 , certains opposants à la libéralisation de la loi attendaient un signal sans équivoque en faveur du libéralisme, ces nouvelles pétitions le leur fournissent. On doit ajouter à ces pétitions les nombreuses demandes spéciales qui sont parvenues à la législature ${ }^{109}$.

Le 22 juin 1869, la première loi canadienne est adoptée. L'Acte concernant les brevets d'inventions prévoit que tout citoyen peut obtenir un brevet du Canada à condition d'y avoir résidé au moins un an au moment de la demande. Une clause de fabrication obligatoire est inscrite: les inventeurs ont trois ans pour mettre leur invention en exploitation au Canada. Les brevets octroyés au Canada avant l'adoption de cette loi et appartenant à des sujets britanniques ayant résidé au Canada lors de leurs demandes, peuvent faire étendre leurs brevets à tout le Canada ${ }^{110}$.

106. $D H C$ (1869), 226, 515-520, 583-590, 619-624 et 646.

107. McDougall lit un télégramme de la Chambre de Commerce de New York; il y constate, sur une liste établie par le général Wallbridge et qui énumère des secteurs pour lesquels la réciprocité paraît souhaitable, que l'importance de la réciprocité des brevets est telle qu'elle occupe le deuxième rang sur cette liste. Voir ibid., 4 juin 1869, 620.

108. Une trentaine de pétitions, avec au-delà de 500 signatures, sont présentées à chacune des chambres de la législature, dont celle provenant de la Charles Legge \& company et de la Chambre des Arts et Manufactures du Bas-Canada. Voir ANQ, 06M-P82.2, Chambre des Arts et Manufactures du Bas-Canada, Correspondance 1857-1873, $1^{\text {er }}$ mai 1869; JHC; (1869), 95, $103,112,123,138,161,170,197$ et $234 ; J S(1869), 72,82,88,104,113,118,130,133,147$, 154, 167 et 195; RG17 AI1, vol. 35, dossiers $3184,3198,3200,3207,3216,3225,3227$ et 3229 ; RG17 AI1, vol. 36, dossiers 3262 et 3343 et Denis Veilleux, op. cit., 131.

109. Elle en reçoit 4 en 1867-1868 et dix autres en 1869 .

110. $32-33$ Vict c. 11 . 
Cette loi est un pas timide vers le libéralisme en matière de brevets - les Américains peuvent obtenir des brevets canadiens même s'ils doivent pour cela se soumettre à certaines contraintes - et elle est certainement le fruit d'un compromis nécessaire entre les différentes lois provinciales. Mais tout cela n'allait pas faire taire les partisans du libéralisme.

\section{LA VICTOIRE DES PARTISANS DU LIBÉRALISME - EN MATIÈRE DE BREVETS}

En juillet 1869, Scientific American évalue que la nouvelle loi canadienne est un pas en avant malgré le fait que les inventeurs américains devront remplir des conditions difficiles pour faire respecter leurs droits au Canada ${ }^{111}$. Puis, au début d'octobre, la revue répond à un article ayant paru dans le Ottawa Times, qui défendait le protectionnisme canadien en matière de brevets en invoquant la jeunesse et le faible développement économique du pays. La revue américaine admet que la nouvelle loi est meilleure que l'ancienne (celle de la Province du Canada sans doute), mais que la situation des inventeurs américains n'est pas changée. Pour Scientific American, les Canadiens veulent simplement voler les inventions des étrangers ${ }^{112}$.

La revue américaine poursuit, au début de 1870, sa campagne en faveur d'une libéralisation au Canada en faisant connaître à ses lecteurs une protestation présentée au Secrétaire d'État aux Colonies à Londres, probablement rédigée par des inventeurs britanniques. La revue exigeait que la nouvelle loi canadienne soit rejetée «on the grounds that it deprives the subjects of the Crown of their equal rights throughout the Empire». Bien que l'article déplore le fait qu'encore une fois les Américains sont laissés pour compte alors qu'ils contribuent au progrès du Canada ${ }^{113}$, il se réjouit néanmoins de cette protestation qui vient appuyer les doléances américaines.

Le gouvernement anglais fera donc connaître sa déception envers la nouvelle loi canadienne. Dans une lettre du 24 février 1870, le Secrétaire d'État aux Colonies prévient le gouverneur général du Canada que la reine n'exercera pas son droit de refus de la nouvelle loi canadienne. Mais il ajoute qu'il est déçu du sort réservé aux inventeurs britanniques ${ }^{114}$.

Au Canada, les forces libérales mettent peu de temps à réagir à l'adoption de la loi des brevets de 1869 et à ajouter leurs pressions à

111. $S A, 21,3$ (17 juillet 1869$): 39$.

112. Ibid., 21,14 (2 octobre 1869): 213.

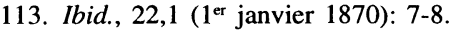

114. ANC, RG17 AI1, vol. 36, dossier 3307. 
celles venant de l'étranger. Entre février et avril 1870, onze pétitions sont envoyées au ministre de l'Agriculture ${ }^{115}$. D'autres pressions, individuelles celles-là, sont adressées au gouvernement ${ }^{116}$.

Les pétitions demandent une loi libérale dans laquelle quiconque peut obtenir un brevet canadien à la seule condition de manufacturer au Canada l'invention pour laquelle un brevet est obtenu. On demande également que les Canadiens puissent breveter les inventions étrangères que leurs créateurs ne veulent pas breveter eux-mêmes au Canåda, ce que l'on appelle des brevets d'introduction. Plusieurs raisons sont invoquées pour justifier cette position. Elles tournent toutes autour d'un argument central selon lequel le développement industriel du Canada a besoin d'une loi libérale et que ce développement industriel ferait prospérer grandement l'économie canadienne dans son ensemble.

À toutes ces pressions s'ajoute l'adoption de la loi américaine de juillet 1870. Cette nouvelle loi accordait désormais à quiconque le droit d'obtenir des brevets aux les mêmes tarifs que ceux exigés des inventeurs américains. Il n'y avait donc plus aucune contrainte à respecter en ce qui a trait à la résidence, à la nationalité ou à l'obligation de manufacturer. Les États-Unis passaient d'une loi libérale basée sur le principe de réciprocité à une loi libérale tout court. Cette fois, les intérêts des inventeurs canadiens aux États-Unis sont garantis. $\mathrm{Ce}$ changement allait ébranler les positions plus ou moins protectionnistes du gouvernement canadien, quoiqu'en 1870, aucun projet de loi visant à donner à la loi des brevets un caractère plus libéral ne soit présenté à la législature canadienne.

De nouvelles pétitions, onze en tout, furent adressées à la Chambre des communes et au Sénat au cours des mois de février et de mars 1871. Une de ces pétitions vient de la Chambre de commerce du Canada. Elle demande que les sujets britanniques ne soient pas dans l'obligation d'avoir résidé pendant au moins un an au Canada. Les autres pétitions demandent simplement l'élimination de l'obli-

115. Ces pétitions sont présentées imprimées et toutes semblables au bas desquelles sont apposées plus de 550 signatures. ANC, RG17 AI1, vol. 35, dossiers 3184, 3198, 3200, 3207, $3216,3225,3227$ et 3229 ; vol. 36, dossiers 3262 et 3229 ; JHC $(1870), 32,55,66,82,97,132$, 141 et $210 ; J S(1870), 34,40,41,43,56,59,83,86,88$ et $108 ; D H C(1870), 279,335$ et 1870 et Denis Veilleux, op. cit., 145-146.

116. Richard Lewis, dans un mémoire présenté au ministre de l'Agriculture, demande d'enlever de la loi canadienne la disposition restrictive quant à la résidence d'au moins un an au Canada comme condition d'obtention de brevets. Un des éléments apportés par Lewis pour justifier sa position est à l'effet que le gouvernement canadien cherche à obtenir le renouvellement du Traité de réciprocité alors que sa loi des brevets est basée sur un principe bien loin de celui de la réciprocité. Pour Lewis, cela est contradictoire et risque de nuire au succès des négociations pour le renouvellement du Traité. Voir ANC, RG17 AI1, vol. 36, dossier 3276. 
gation de résidence pour tous ${ }^{117}$. Pendant que la législature reçoit ces pétitions, un projet de loi est déposé le 27 février à la Chambre des communes par Thomas Oliver, député de Oxford-Nord en Ontario. Le projet prévoit l'abolition de la nécessité d'avoir résidé au moins un an au Canada avant de pouvoir obtenir un brevet.

Le ministre de l'Agriculture, Christopher Dunkin, s'oppose à cette abolition. Ses arguments sont à l'effet que, d'une part, le gouvernement n'est pas prêt à légiférer sur cette question et que, d'autre part, l'abolition demandée se trancherait par une invasion de brevets américains au Canada alors qu'il y aurait déjà assez, selon eux, de brevets accordés. Le projet d'Oliver sera retiré ${ }^{118}$.

Nous avons vu que le gouvernement du Canada cherchait à utiliser la possibilité de changer la nature protectionniste de la législation canadienne en matière de brevets comme monnaie d'échange afin d'obtenir le renouvellement du Traité de réciprocité. En 1869, le gouvernement conservateur était allé aussi loin que possible pour satisfaire les tenants du libéralisme, tout en maintenant une loi assez protectionniste pour conserver un élément de négociation. Mais en 1871, cinq ans après la fin du Traité signé en 1854, l'espoir d'un renouvellement est faible. La signature du Traité de Washington en 1871 règle la question des droits de pêche sur la côte atlantique, ce qui élimine un élément de plus favorisant un accord éventuel de libreéchange. Finalement, les Américains n'ont jamais vraiment voulu au $\mathrm{XIX}^{\mathrm{e}}$ siècle renouveler le Traité de réciprocité canado-américain, une forte tendance protectionniste en matière commerciale s'y étant développée ${ }^{119}$.

De plus, les Américains n'étaient toujours pas satisfaits de la loi canadienne sur les brevets. Dans son rapport annuel de 1872, le Commissaire des brevets des États-Unis rappelle la source du mécontentement des manufacturiers américains:

The inventor, for instance, of a process or a machine for making a given article of manufacture, may suffer by the practice of the invention across the boundary line between the two countries by parties who furnish the manufactured article in our market at such rates as to be ruinous to the inventor. There can be no protection against this practice except in cases in which a patent can be

117. $J H C(1871), 29,48,56,64$ et $135 ; J S(1871), 47,52,56,61$ et 75 et Denis Veilleux, op. cit., 147-148.

118. $D H C$ (1871), 141 et 827-829.

119. Voir D. C. Masters, La réciprocité (Ottawa, La Société historique du Canada, 1973), brochure $\mathrm{n}^{\circ} 12$, et Pierre Trudel, Le traité de réciprocité 1854 (Ottawa, Les Éditions de l'Université d'Ottawa, coll. "Cahiers d'histoire», $\left.\mathrm{n}^{\circ} 1,1968\right)$. 
obtained on the article of manufacture itself as well as upon the process or upon the machine by which it is made. But where the article itself is old, and therefore not patentable, the inventor of the new process or machine for making it is, by the means indicated, left practically without protection ${ }^{120}$.

Le Commissaire propose que des négociations soient amorcées entre son gouvernement et celui du Canada.

Devant ces pressions, et alors que les négociations pour un nouveau Traité de réciprocité étaient à toutes fins pratiques vouées à l'échec, le gouvernement canadien n'avait plus d'autre choix que de se laisser guider par les influences canadiennes en matière de brevets. Or, le libéralisme semblait dominer depuis 1869. Le ministre de l'Agriculture faisait d'ailleurs état de cette situation dans son rapport annuel de $1872^{121}$.

En avril et mai 1872, la législature canadienne reçoit de nouvelles pétitions, une trentaine, demandant toutes une loi permettant aux étrangers d'obtenir des brevets canadiens ${ }^{122}$. Au même moment, des membres de la Chambre des communes et du Sénat, comme Sanborn, demandent que le gouvernement présente un projet afin d'amender la loi sur les brevets. Un projet est effectivement présenté à la Chambre des communes au début du mois de mai par J. H. Pope, le nouveau ministre de l'Agriculture. Le projet prévoit comme seul changement à la loi de 1869, la possibilité pour tous d'obtenir des brevets canadiens et de rallonger d'un an la période pendant laquelle les inventions doivent commencer à être fabriquées au Canada. Le projet de loi prévoit, par contre, que seules les inventions ayant été brevetées ailleurs dans les douze mois avant la demande au Canada pourraient être brevetées au Canada ${ }^{123}$.

Cette dernière disposition visait à protéger les industries canadiennes qui utilisaient des technologies étrangères depuis plus d'un an. Seule cette disposition a fait l'objet de débats. Certains députés voulaient que la période soit plus longue pour favoriser les inventeurs étrangers qui s'étaient fait pirater leurs inventions par des Canadiens. Mais le projet de loi ne sera pas amendé. Un député dira d'ailleurs qu'il y a des limites au libéralisme. D'autres opineront que malgré

120. Annual Report of the Commissioner of Patents for the year 1871 (Washington, 1872), 13.

121. «Annual Report», $S P(1872), \mathrm{n}^{\circ} 2 \mathrm{~A}$.

122. $J H C(1872), 19,31,40,57,66,80,82,99$ et 109 ; $J S(1872), 32,35,40,43,46,48$, 51, 53, 59 81, 86 et 92; et Denis Veilleux, op. cit., 151-152.

123. $D H C$ (1872), 394 et 671 ; et $D S$ (1872), 59. 
tout, c'est-à-dire malgré le virage libéral, il faut chercher avant tout à protéger les manufactures canadiennes ${ }^{124}$.

Le projet de loi, l'Acte concernant les Brevets d'inventions sera finalement adopté et sanctionné le 14 juin $1872^{125}$, quelques mois avant les élections prévues pour l'automne.

\section{CONCLUSION}

Plusieurs inventeurs ou détenteurs de brevets sont intervenus directement dans les débats opposant protectionnistes et libéraux, d'autres sont intervenus indirectement, en passant par les Chambres des Arts et Manufactures par exemple. Mais il ne faudrait pas passer sous silence l'influence que l'augmentation du nombre de brevets peut avoir exercé. En effet, au cours de la période 1852-1872, le nombre de brevets octroyés a augmenté d'une manière significative. Par exemple, au Québec et en Ontario, il est passé de près de 200 en 1867 à plus de 500 en 1869. Au Nouveau-Brunswick, ce nombre s'est accru d'une moyenne de 10 par année, entre 1852 et 1866 , à une moyenne de 140 , entre 1866 et 1869 . Le nombre de détenteurs de brevets a fait une progression semblable. Le poids politique des inventeurs s'est donc considérablement renforcé précisément et particulièrement dans une période critique $^{126}$. Cela est d'autant plus important que plusieurs inventeurs sont liés de près ou de loin à des personnalités politiques et aux secteurs industriel et commercial qui connaissaient déjà une croissance notable ${ }^{127}$.

De la même façon, le nombre de brevets américains détenus par des Canadiens augmente sensiblement durant la période, indiquant une volonté grandissante des inventeurs canadiens d'élargir leur marché. Cette tendance favorisait bien sûr leur adhésion au courant libéral en matière de brevets. Alors qu'un seul brevet est accordé à un Canadien aux États-Unis en 1852, 10 sont accordés en 1867, 16 en 1869 et près de 150 en 1872, après que les restrictions contre les étrangers aient été éliminées ${ }^{128}$.

124. DHC (1872), 671 et 1041-1044.

125. 35 Vict. c. 26

126. Il faut noter que le nombre de brevets octroyés est indiqué dans chacun des rapports annuels du ministre de l'Agriculture entre 1860 et 1872 . Pour plus de détails, voir Denis Veilleux, op. cit., 133-138.

127. List of Canadian Patents (...), Lovell's Canadian Dictionary for 1871 (...), Patents of Canada from 1849 to 1855 (Toronto, 1860); JBAMUC (1861-1867); The Year Book and Almanac of Canada, 1867-1869; Patents of Canada from 1824 to 1849 (Toronto, 1860); The Canadian Patent Office Record (1873); et ANC, MG9 A1, New Brunswick, Executive Council, Patents of Invention, vol. 123, finding aid $\mathrm{n}^{\circ} 121$.

128. Annual Report of the Commissioner of Patents (Washington, 1852, 1867 et 1869); et Official Gazette of the United States Patent Office (1872). 
Si les inventeurs ont sans doute joué un rôle important dans le mouvement de libéralisation, il est certain que les facteurs conjoncturels ont joué parfois à l'encontre et parfois en faveur de ce mouvement. Ainsi, la possibilité d'une confédération des colonies de l'ANB a retardé l'adoption d'une loi plus libérale. Inversement, la création de la Confédération a fourni un terrain propice à l'adoption d'une telle loi puisqu'elle induisait la recherche d'un compromis entre des lois provinciales passablement divergentes. En ce qui concerne le Traité de réciprocité, plus il était improbable de le voir renouveler, meilleures étaient les chances de voir adopter une loi des brevets plus libérale.

L'historien R. T. Naylor a interprété la loi des brevets de 1872 comme un moyen pris par le gouvernement de l'époque pour inciter les Américains à investir dans l'économie canadienne accentuant ainsi sa dépendance des États-Unis ${ }^{129}$. Cependant, cette interprétation fait peu de cas de la diversité des intérêts qui se sont affrontés. En effet, nous avons montré que des forces sociales de plus en plus organisées, agissant dans une conjoncture devenant progressivement favorable, et non la seule volonté du gouvernement, sont à la source de l'adoption de la loi de 1872. En fait, la libéralisation des lois des brevets entre 1852 et 1872 au Canada met moins en évidence une politique consciente et délibérée des gouvernements canadiens qu'une réponse aux influences des inventeurs et des manufacturiers, couplée aux pressions étrangères, notamment américaines.

Ceci dit, notre analyse ne remet pas en question le fait qu'une fois adoptée cette loi ait effectivement accentué la dépendance technologique du Canada à l'égard des États-Unis. Mais cela est une autre question. Ce qu'il faut retenir ici, c'est que cette dépendance a été le fruit du courant libéral en matière de brevets, que l'on doit certainement comprendre comme un sous-ensemble de l'idéologie libérale qui se faisait plus influente.

129. Effectivement, le nombre de brevets détenus par des Américains a considérablement augmenté après l'adoption de la loi sur les brevets de 1872. Ce nombre a d'ailleurs largement dépassé celui des brevets détenus par les Canadiens. Cette augmentation, et le contrôle sur le développement technologique et économique qu'elle peut entraîner, est un des éléments à l'appui de la thèse de la dépendance accrue de l'économie canadienne. Pour l'évolution du nombre de brevets accordés selon l'origine des demandeurs, voir les rapports annuels du commissaire des brevets du Canada. Pour la thèse dont il est question, voir Naylor, op. cit. 